\begin{tabular}{|c|c|}
\hline Title & Spontaneous flow reversals in Rayleigh-Bénard convection of a liquid metal \\
\hline Author(s) & Y anagisawa, Takatoshi; Y amagishi, Y asuko; Hamano, Y ozo; Tasaka, Y uji; Takeda, Y asushi \\
\hline Citation & $\begin{array}{l}\text { Physical Review E, 83(3), } 036307 \\
\text { https://doi.org/10.1103/PhysRevE.83.036307 }\end{array}$ \\
\hline Issue Date & 2011-03 \\
\hline Doc URL & http:/hdl.handle.net/2115/45148 \\
\hline Rights & (2011 A merican Physical Society \\
\hline Type & article \\
\hline File Information & PRE83-3_036307.pdf \\
\hline
\end{tabular}

Instructions for use 


\title{
Spontaneous flow reversals in Rayleigh-Bénard convection of a liquid metal
}

\author{
Takatoshi Yanagisawa, ${ }^{*}$ Yasuko Yamagishi, and Yozo Hamano \\ Institute for Research on Earth Evolution, Japan Agency for Marine-Earth Science and Technology, Yokosuka, Japan \\ Yuji Tasaka and Yasushi Takeda \\ Graduate School of Engineering, Hokkaido University, Sapporo, Japan \\ (Received 6 October 2010; revised manuscript received 3 December 2010; published 14 March 2011)
}

\begin{abstract}
We report a finding of spontaneous flow reversals of roll-like patterns in liquid gallium Rayleigh-Bénard convection. The vessel has a square geometry with an aspect ratio of 5, and a horizontal magnetic field is applied to align the rolls. The flow patterns were visualized by ultrasonic velocity measurements, and the processes of the reversal were clearly observed. The basic flow pattern observed in the vessel is a four-roll structure with its axis parallel to the magnetic field. Emergence of a new circulation at a corner of the vessel causes flow reversal with reorganization of the whole pattern. The flow keeps relatively steady four-roll structure for most of the duration, while the reversal of it is over in a short time. The reversals of the flow occur randomly with the interval time between reversals being much longer than the circulation time.
\end{abstract}

DOI: 10.1103/PhysRevE.83.036307

PACS number(s): 47.20.Bp, 47.27.De, 47.65.-d

\section{INTRODUCTION}

The nature of flow structures in Rayleigh-Bénard (R-B) convection and an understanding of the mechanism of generation are important problems (e.g., $[1,2])$, and "flow reversal" in R-B convection has been an active topic in this decade (see recent review in [3]). The occurrence of spontaneous reversals of the mean flow direction in turbulent thermal convection is established, with the observed reversals not being periodic but rather occurring at irregular intervals, with the time scales being much longer than the turnover time of the convection [4,5]. These features provide important evidence that helps in the understanding of a range of phenomena on the Earth, including wind directions in the atmosphere and geomagnetic field reversals.

Most experimental investigations on flow reversals are made in cylinders or rectangular boxes with small aspect ratios $(\leqslant 1)[4-10]$; here the flow pattern is basically a single circulation and is strongly restricted by the sidewalls of the vessels. Experiments with larger aspect ratio geometries would be helpful to understand whether flow reversals can occur in situations with several horizontal coexisting circulations. The working fluids in the reported experiments have mainly been water and gas, whose Prandtl numbers (Pr) are around 1. Liquid metals $\left(\operatorname{Pr} \sim 10^{-2}\right)$ have been used in some experiments [11-13], but obvious flow reversals have not been established with liquid metals. Recently direct measurements of velocity fields of water have been made by particle imaging velocimetry (PIV) [7-10]. These direct measurements of the flow fields have provided much information on details of flow reversals, but PIV cannot be applied for liquid metals because the metals are opaque. The use of liquid metals, none the less, has two major advantages: first, the low Pr makes it relatively easy for the flow to become turbulent, and hence the Ra necessary for flow reversals may be lower than that for fluids with $\operatorname{Pr} \sim 1$. Second, the high electrical conductivity means that external magnetic fields can enhance the two-dimensionality of the

*yanagi@jamstec.go.jp convective flow structure. Further, the study of liquid metal convection in the presence of a magnetic field is important to understand the dynamics of planetary cores. In liquid metals, direct measurements of the spatiotemporal flow field of thermal convection have been realized by the ultrasonic velocity profiler (UVP) method [14]. Applications of the UVP to R-B convection with liquid metals have shown flow patterns with fluctuations $[12,13,15,16]$.

In the present paper, we report flow reversals observed in liquid gallium, where the flow velocity is directly measured by UVP, in a square vessel with an aspect ratio of 5 . We applied a uniform horizontal magnetic field to the convection system. The nondimensional parameters to describe a R-B setting are the Rayleigh number $\left(\mathrm{Ra}=\alpha g \Delta T L^{3} / \kappa \nu\right)$ and the Prandtl number $(\operatorname{Pr}=\nu / \kappa)$, with $\alpha$ being the volume coefficient of thermal expansion, $g$ the gravitational acceleration, $\Delta T$ the vertical temperature difference across the fluid layer, $L$ the fluid layer thickness, $\kappa$ the thermal diffusivity, and $v$ the kinematic viscosity. The effect of the applied magnetic field is characterized by the Chandrasekhar number $(Q=$ $\left.\sigma B^{2} L^{2} / \rho v\right)$ and the magnetic Prandtl number $(\operatorname{Pm}=v / \eta)$, with $\sigma$ being the electrical conductivity, $\rho$ the fluid density, $B$ the intensity of the applied magnetic field, and $\eta$ the magnetic diffusivity of the fluid. The aim of the paper is to describe the flow pattern in R-B convection of liquid gallium with reversals and to compare the characteristics of the reversals with the results of $\operatorname{Pr} \sim 1$ fluids.

\section{APPARATUS AND METHODS}

Figure 1 shows the vessel for the liquid gallium convection and the Helmholtz coil system with the positions of the lines for the velocity measurements. The top and bottom plates are made of copper, and the temperature of each plate is maintained by circulating water. The four sidewalls of the vessel are 10-mm-thick Teflon, with electric conductivity much lower than gallium, and may be considered as a perfect insulator. The inside measurements of the vessel are $200 \mathrm{~mm}$ square and $40 \mathrm{~mm}$ deep $(L)$, giving an aspect ratio of 5 (length 
(a)
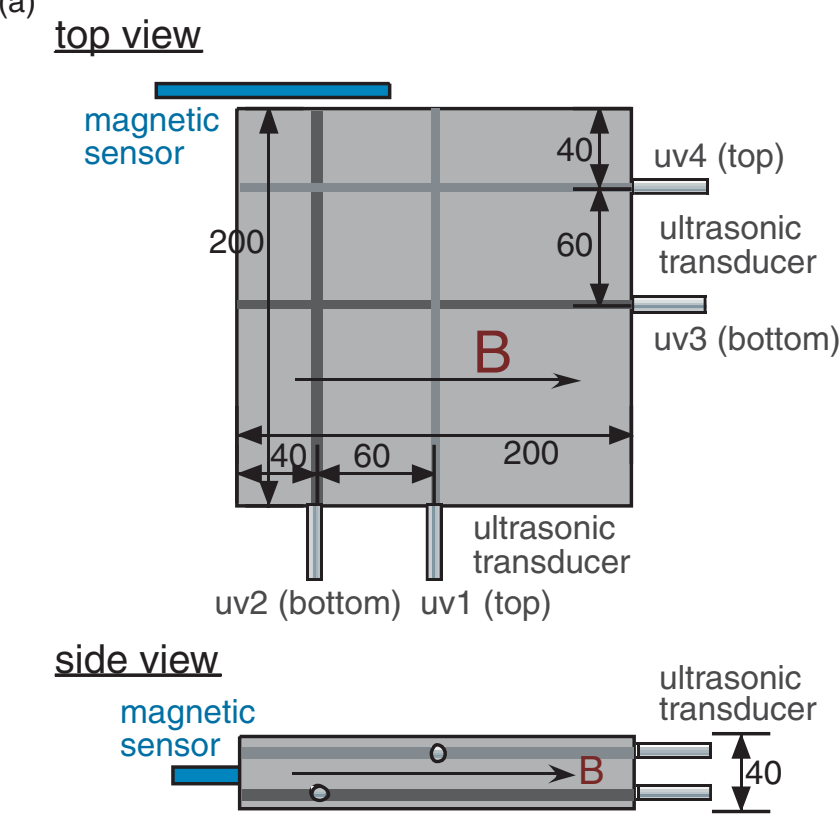

(b)

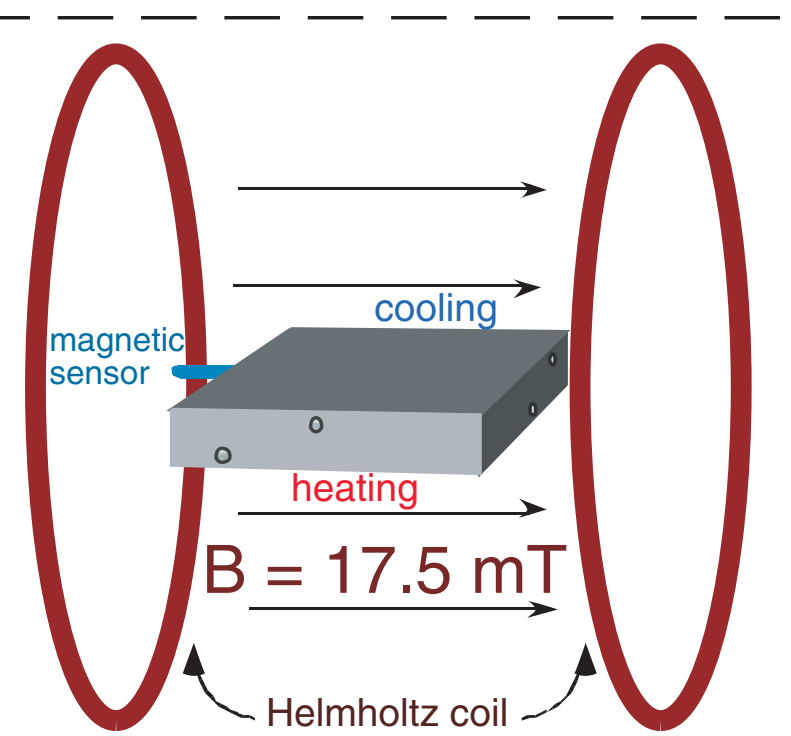

FIG. 1. (Color online) (a) Top and side views of the vessel. The numbers are the dimensions in $\mathrm{mm}$. Darker gray lines in the vessel indicate the ultrasonic beam lines for flow velocity measurements near the bottom, and the brighter gray lines indicate the ultrasonic beam lines for flow velocity measurements near the top, $10 \mathrm{~mm}$ from the top or bottom plate. The lines uv1 and uv2 measure the velocity profiles perpendicular to the applied magnetic field $B$, while uv3 and uv4 are parallel to $B$. (b) The 600 -mm-diameter Helmholtz coil and the vessel in the horizontal magnetic field.

to height). The direction of the applied magnetic field is horizontal and is parallel to one side of the vessel as shown in Fig. 1. Ultrasonic transducers for the UVP are set in holes in the Teflon sidewalls and are in direct contact with the liquid gallium. The flow velocities of the gallium were measured along four lines from the ultrasonic transducers (uv1uv4); these four transducers were switched on in order for 1.25 -s periods, and the sampling for the lines took place at 5-s intervals. The UVP measures the projected flow velocity along each line. The Rayleigh number was controlled by varying the temperature difference between the top and bottom plates $(\Delta T)$, and the intensity of the applied magnetic field (B) was maintained at $17.5 \mathrm{mT}$ throughout the experiments here, giving the Chandrasekhar number $Q=1.0 \times 10^{3}$. The $\mathrm{Pr}$ and Pm of gallium are 0.025 and $1.5 \times 10^{-6}$, respectively. Further details of the experimental procedure are provided elsewhere [15-17].

\section{RESULTS}

We observed spontaneous flow reversals at $\mathrm{Ra}=1.5 \times 10^{4}$ with $\Delta T=0.71 \mathrm{~K}$, and details of the case are presented here. Figure 2 shows velocity profiles observed at the four lines (uv1-uv4) for $19500 \mathrm{~s}(\sim 5.4 \mathrm{~h})$. The direction and magnitude of the velocity are shown in color, blue (minus) shows flow toward each transducer and red (plus) shows flow away from each transducer. The initial state $(t=0 \mathrm{~s})$ was a uniform temperature with the $17.5-\mathrm{mT}$ horizontal magnetic field. The vertical temperature difference was set at $300 \mathrm{~s}$, and four rolls of convection pattern emerged at $\sim 400 \mathrm{~s}$. The convection pattern is clearly affected by the applied magnetic field, and the flow shows anisotropic structures. One noteworthy feature of these profiles is the larger velocities observed in uv1 and uv2 $(\sim 5 \mathrm{~mm} / \mathrm{s})$ than in uv3 and uv4 ( $\sim 2 \mathrm{~mm} / \mathrm{s})$. In the 400-1200 s period, four clusters of velocities are observed with opposite signs in uv1 and uv2, which suggests the existence of a nearly two-dimensional roll-like structure with the axis parallel to the direction of the applied magnetic field. Around $1200 \mathrm{~s}$ the four-roll pattern starts to lose stability and later the pattern shows irregular reversals of the flow direction throughout the remaining duration of the experiment.

One flow structure dominantly observed in the experiment is the four-roll pattern with blue (red) patches around 0-50 mm in uv1 (uv2), which shows downwelling flows at both sidewalls and the center as indicated in Fig. 3(b). The other dominant flow structure is also a four-roll pattern with the flow direction opposite to this [Fig. 3(a)]. The fraction of time that the flows show either of these four-roll states is relatively large, and the transition between the two patterns occurs over a shorter time than the steady four-roll state is maintained. The transition proceeds as follows: a small circulation emerges at a corner of the vessel in either sidewall. It grows up to the fifth roll and expands horizontally for a while; then the global flow reverses with a reorganization of the whole pattern. In Fig. 2, both the propagation of patterns (tilted sections) and the reorganization of the flow direction (at the discontinuities) are observed in uv1 and uv2. The experiment here showed 22 times of global reversals as indicated by the arrows at the top of Fig. 2, giving an average time between intervals of $\sim 800 \mathrm{~s}$. As the length of circulation of a roll is $\sim 180 \mathrm{~mm}$ and the typical flow velocity is $\sim 5 \mathrm{~mm} / \mathrm{s}$, the circulation time of the flow for a roll is 30-40 s. This suggests that the average intervals of the flow reversal are much longer than the circulation time. The average time interval is also longer than the thermal diffusion time for the layer $\left(L^{2} / \kappa \sim 136 \mathrm{~s}\right.$ with $\left.\kappa=1.2 \times 10^{-5} \mathrm{~m}^{2} / \mathrm{s}\right)$. The profiles in uv 3 and uv4 show smaller and more random fluctuations around zero velocity. The vertical stripes in uv3 and uv4 indicate the existence of vessel-scale coherent flow patterns, 


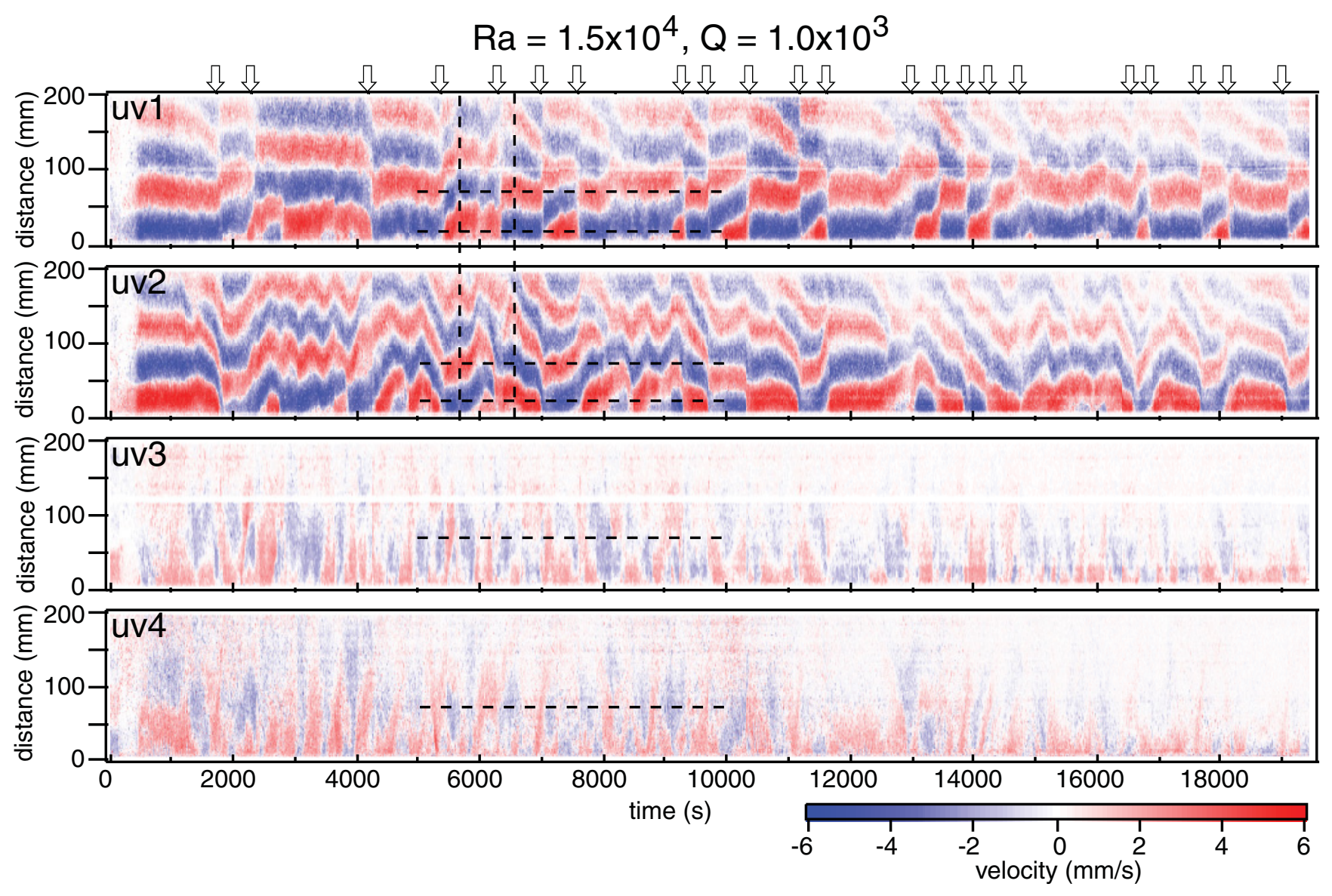

FIG. 2. (Color) Flow velocity profiles observed by the four lines uv1-uv4. Ra $=1.5 \times 10^{4}, Q=1.0 \times 10^{3}$. The vertical axis shows the distance from the transducers, with $200 \mathrm{~mm}$ the opposite side of the vessel. The direction and magnitude of the horizontal velocity is shown in color: blue (minus) shows flow toward the transducer and red (plus) is flow away from the transducer. The horizontal axes show the time elapsed in the experiment. A four-roll structure with axis parallel to the magnetic field is dominant, and the profiles show many reversals of flow direction with nonperiodic intervals. The arrows at the top indicate the points of global flow reversals. The vertical broken lines at 5700 and $6600 \mathrm{~s}$ indicate the times of the slices shown in Fig. 3, and the horizontal broken lines at 20 and 70 mm indicate the positions and range of the time series in Fig. 4.

though the velocities are small. These patterns also change flow directions, but with shorter time scales than observed in uv1 and uv2. There is no clear correlation between the flow patterns parallel (uv3, uv4) and perpendicular (uv1, uv2) to the directions of the magnetic field.

Figure 3 displays two time-slices from the velocity data, with the inferred flow structures by assuming twodimensionality of the flow. Figures 3(a) and 3(b) show the four-roll patterns dominantly observed in the present experiment, and the amplitudes of the flow velocities in these two states have similar values. The slight differences in the zero-crossing position of the velocity profiles between uv1 and uv2 indicate the vertical tilt of the rolls as shown in the schematic views. This may arise from the existence of the vessel-scale circulation in this plane, because the directions of the tilt are common among the rolls. In the transitions between these two patterns, the three-dimensionality of the flow structure may be important.

Figure 4 shows the temporal variations in the velocities from 5000 to $10000 \mathrm{~s}$ at six fixed positions in the vessel (both near the top and bottom: (a) $20 \mathrm{~mm}$ and (b) $70 \mathrm{~mm}$ distance from the transducer side in the perpendicular direction to $B$; (c) $70 \mathrm{~mm}$ in the parallel direction to $B$ ). These graphs indicate how the reversals of the flow are observed at specific points. When the four-roll pattern exists, the positions (a) and (b) are close to the center of the first and second roll. In Figs. 4(a) and 4(b), the profiles of uv 1 and uv 2 are approximately symmetric about the zero velocity for a large fraction of the duration; this is because the measurement lines are symmetric around the middle of the vessel (uv1 is near the top and uv2 is near the bottom) and observe the upper or lower parts of the same roll. The four-roll state is characterized by a nearly constant velocity of $\pm 5 \mathrm{~mm} / \mathrm{s}$, and the time necessary for the reversals is much shorter than the durations of the four-roll states. Six reversals of the global flow structure are identified as indicated by the arrows in Fig. 2 in the range of these time series. The global reorganizations occurred at around 5400, 6300, $7000,7600,9300$, and $9700 \mathrm{~s}$, which can be recognized as the positions where red changes to blue and vice versa in Fig. 2, and are characterized by abrupt changes of sign in uv1 or uv 2 in Figs. 4(a) and 4(b). The slower increases and decreases in the velocity in Figs. 4(a) and 4(b) correspond to the tilted 
(a) $5700 \mathrm{~s}$

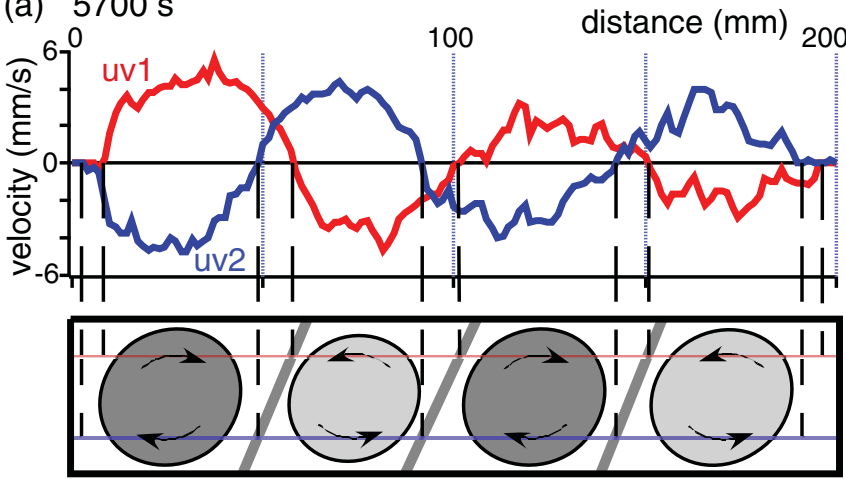

(b) $6600 \mathrm{~s}$

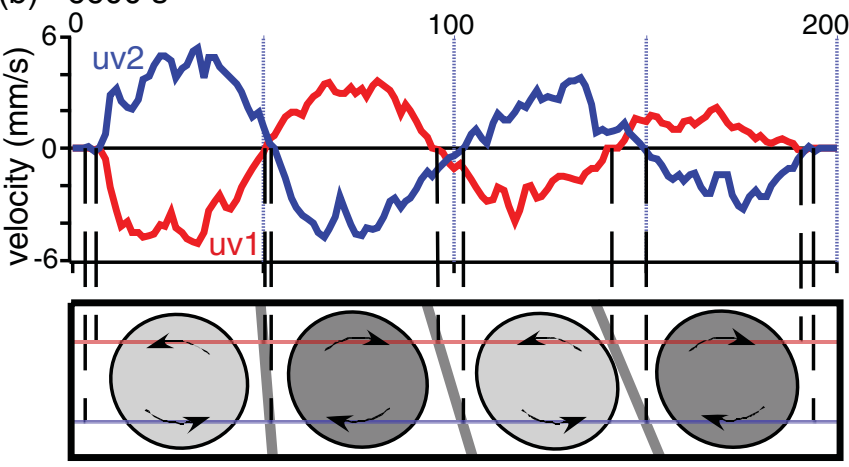

FIG. 3. (Color) Time slices of the velocity data at (a) $5700 \mathrm{~s}$ and (b) $6600 \mathrm{~s}$, with schematic views of the roll patterns. Red (blue) lines are the profiles from uv1 (uv2). A four-roll structure is indicated in both time slices, with the direction of flow perfectly inverted. The tilted gray lines in the schematic views indicate the inferred region of dominance of each roll from the differences in the zero crossing positions for uv1 and uv2 (shown by vertical broken lines). The relatively smaller amplitudes of the velocity in the far side of the transducer are due to the expansion of the ultrasonic beam lines and averaging with smaller flow velocities near the top and bottom plates.

sections in Fig. 2. "Aborted reversals," where the velocity starts to decrease or increase but returns to the original pattern, are also observed in some positions (at around 6000, 8200, and $8700 \mathrm{~s}$ ). The profiles for uv3 and uv4 show a shorter time scale of random fluctuations. Histograms of the velocity values in Fig. 5 display a single peak around zero velocity for uv3 and uv4, while uv1 and uv2 have double peaks around $\pm 5 \mathrm{~mm} / \mathrm{s}$. Double peaks in velocity histograms reflect the bistable state of the flow and are commonly observed for flow reversals with water and gas (e.g., [4]). The profiles for uv3 and uv4 show no clear similarities in appearance with uv1 or uv2, though they correlate at some time points.

We analyzed the time intervals between reversals. The times when the velocity profiles change sign in uv1 were determined for the data from 1500 to $19500 \mathrm{~s}$ at the 20-, 70-, 120-, and $170-\mathrm{mm}$ positions. There are over 100 reversals recognized in this way, some not global but regional, with the time interval $\tau$ ranging from 50 to $2400 \mathrm{~s}$. In Fig. 6, all of these time intervals are sorted in descending order and plotted in the form of a cumulative distribution of a probability density function (PDF) $p(\tau)$ as $P\left(\tau_{1}\right)=\int_{\tau_{1}}^{\infty} p(\tau) d \tau$ (see [5]). The points fit well for $\tau_{1} \leqslant 2000 \mathrm{~s}$ by the relation $P\left(\tau_{1}\right) \sim \exp \left(-\tau_{1} / \tau_{m}\right)$, with (a) $20 \mathrm{~mm}$ from transducer perpendicular to $\mathrm{B}$

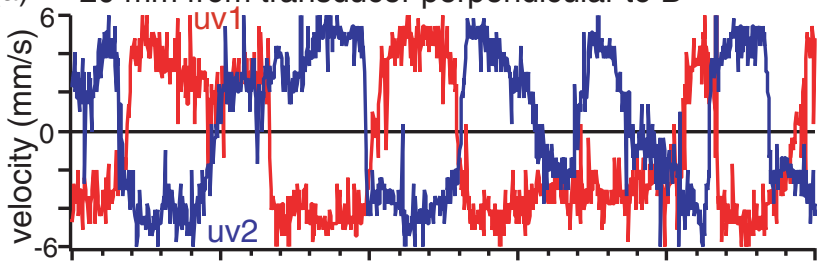

(b) $70 \mathrm{~mm}$, perpendicular to $\mathrm{B}$

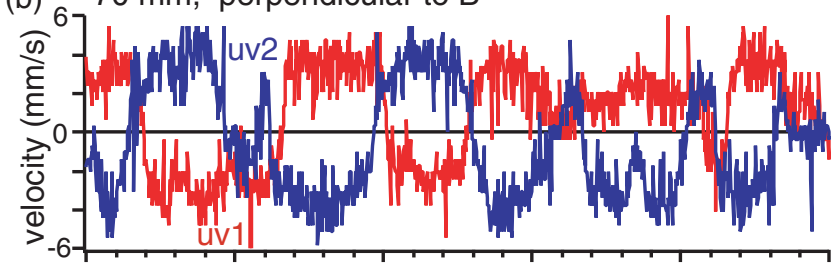

(c) $70 \mathrm{~mm}$, parallel to $B$

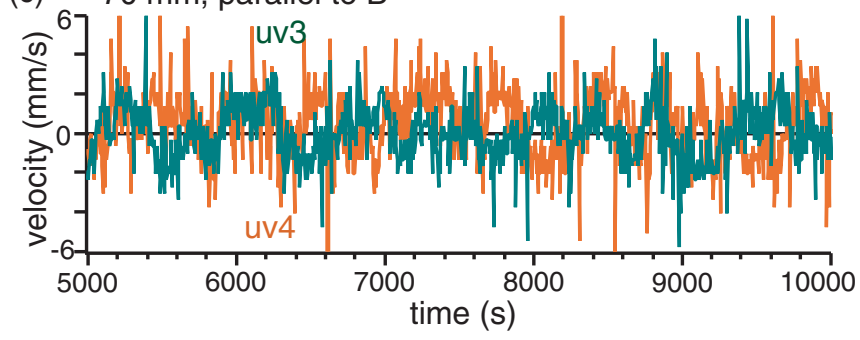

FIG. 4. (Color) Temporal variations of the velocity (uv1-uv4) for $5000-10000 \mathrm{~s}$. (a) $20 \mathrm{~mm}$ from the transducer in the direction perpendicular to $B$ : red, uv1; blue, uv2. (b) $70 \mathrm{~mm}$ in the direction perpendicular to $B$ : red, uv1; blue, uv2. (c) $70 \mathrm{~mm}$ in the direction parallel to $B$ : green, uv3; orange, uv4. The outliers on these velocity profiles are not actual velocities but noise arising from the UVP measurements.

$\tau_{m}=660 \mathrm{~s}$. This indicates that the reversals can be thought of as a Poisson process (randomly occurring, no hysteresis) and with a characteristic time of interval of $660 \mathrm{~s}$.

\section{DISCUSSION}

The present visualization indicates that the flow reversals are caused by the emergence of a new circulation structure at the corner on either sidewall. The newly generated minor

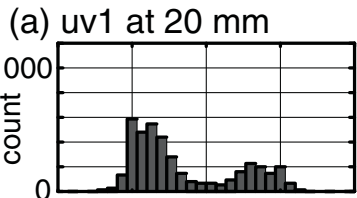

(c) uv3 at $70 \mathrm{~mm}$

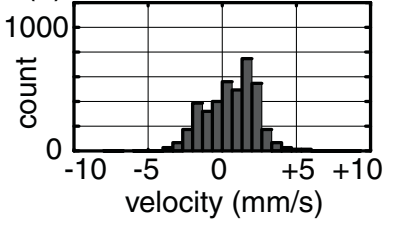

(b) uv2 at $20 \mathrm{~mm}$

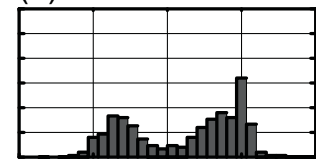

(d) uv4 at $70 \mathrm{~mm}$

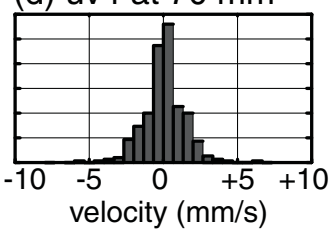

FIG. 5. Histograms of the velocity values observed at four positions, for 1500-19500 s. (a) uv1 at $20 \mathrm{~mm}$ and (b) uv2 at $20 \mathrm{~mm}$, from the transducer in the direction perpendicular to $B$. (c) uv3 at $70 \mathrm{~mm}$ and (d) uv4 at $70 \mathrm{~mm}$, in the direction parallel to $B$. Vertical axis is the number of counts for each velocity range. 


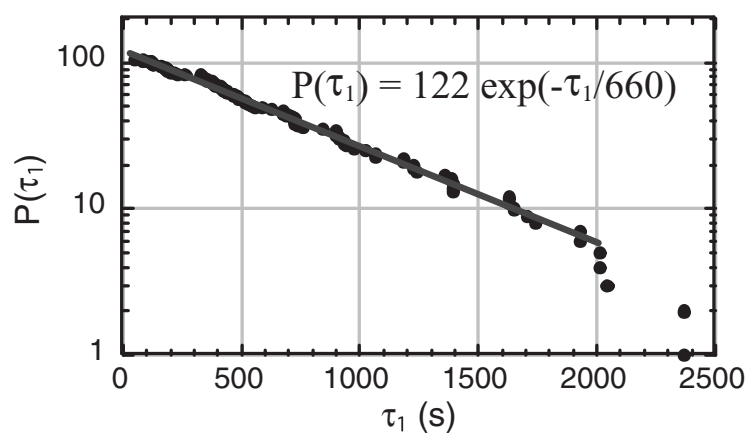

FIG. 6. The cumulative distribution of the interval times for reversals. These are the combined plots at 20,70,120, and $170 \mathrm{~mm}$ from the transducer for uv1. The fitted straight line indicates the exponential distribution with $\tau_{m}=660 \mathrm{~s}$.

circulation structure expands and the roll pattern propagates horizontally; at a certain moment a reorganization of the whole pattern occurs, and the phase of the global pattern is inverted. This may correspond to the emergence of plumes near the corner causing global flow reversals observed by PIV in water $\mathrm{R}-\mathrm{B}$ convection [10]. The feature of the interval times observed in the present experiment, that it can be thought of as a Poisson process with random intervals, is similar to that in [5] for gas convection in a cylindrical geometry. Further, the relation that the characteristic time of intervals is 20-30 times longer than the circulation time of the flow in a roll is consistent with the reported results. These findings may indicate a common mechanism for the flow reversals in the present study with liquid gallium in a horizontally larger vessel and $\operatorname{Pr} \sim 1$ fluid in small aspect ratio geometries.

We performed several experiments with different values of $\Delta T$, under the same value of $B=17.5 \mathrm{mT}$. Figure 7 shows flow velocity profiles for the lower and higher $\mathrm{Ra}$ than the Ra presented in the previous section, with the same value of $Q=1.0 \times 10^{3}$. These are the expanded profiles for $5000 \mathrm{~s}$ from over $10000 \mathrm{~s}$ duration of experiments. In Fig. 7(a), $\mathrm{Ra}=8.5 \times 10^{3}$ with $\Delta T=0.40 \mathrm{~K}$, the observed flow pattern is almost steady with a four-roll structure whose axis is parallel to the magnetic field. These velocity profiles indicate the same structure of roll with its flow direction as schematically shown in Fig. 3(b), and it lasts without reversals. In Fig. 7(b), $\mathrm{Ra}=5.9 \times 10^{4}$ with $\Delta T=2.8 \mathrm{~K}$, strongly time-dependent flow is observed. In this case, the average velocity is larger for uv1 than for uv3, which is the effect of the applied magnetic field, but no clear roll-like structure exists. The flow pattern is broader than the four-roll structure, and it shows a large amplitude of fluctuations. In either cases in Fig. 7, spontaneous flow reversals as shown in Fig. 2 are not observed. These experiments indicate that spontaneous flow reversals occur in a limited range of $\mathrm{Ra}$.

A regime diagram of the convection pattern under a horizontal magnetic field was established in a previous study [17] in relation to $\mathrm{Ra}$ and $Q$ for the ranges $9.3 \times 10^{3} \leqslant \mathrm{Ra}$ $\leqslant 3.5 \times 10^{5}$ and $0 \leqslant Q \leqslant 1.9 \times 10^{3}$, and the flow regime was classified by the interaction parameter $N=\sigma B^{2} L / \rho U$. Here, $N$ is defined by the magnitude ratio of the Lorentz force to the inertial force. The regime diagram in [17] was for a narrow vessel with a 4:1:1 length ratio (1 is the height), (a) $\mathrm{Ra}=8.5 \times 10^{3}, \mathrm{Q}=1.0 \times 10^{3}$

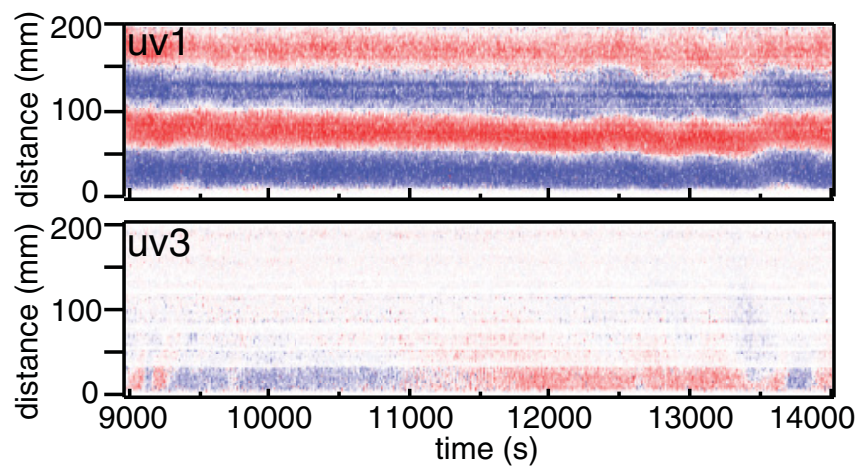

(b) $\mathrm{Ra}=5.9 \times 10^{4}, \mathrm{Q}=1.0 \times 10^{3}$

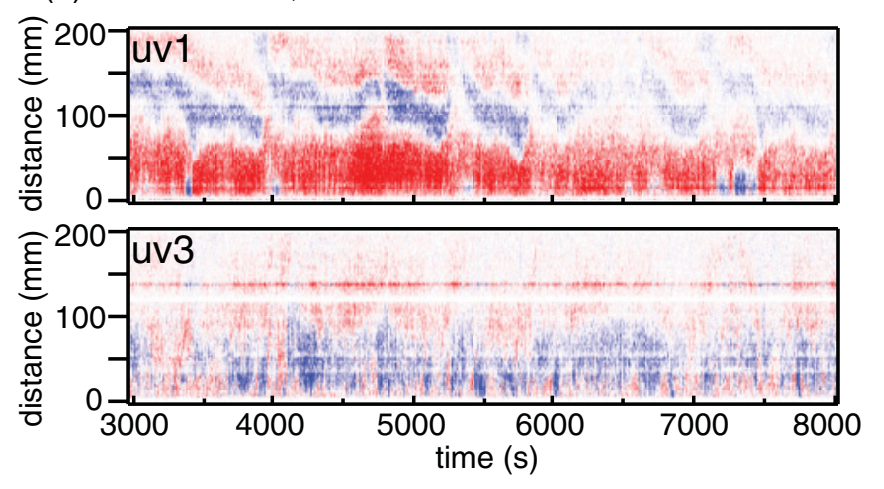

FIG. 7. (Color) Flow velocity profiles observed by the two lines uv1 and uv3, for (a) $\mathrm{Ra}=8.5 \times 10^{3}$ and (b) $\mathrm{Ra}=5.9 \times 10^{4}$. In both cases, $Q=1.0 \times 10^{3}$. The horizontal axes show the time elapsed from the setting of the vertical temperature difference. The color scale is same as that in Fig. 2. (a) Almost steady flow: a four-roll structure with axis parallel to the magnetic field is dominant. (b) Strongly time-dependent flow: the average velocity is larger for uv1 than for uv3, but no clear roll-like structure exists. In either case, flow reversals are not observed.

which may be compared with the present square vessel. It shows that the applied magnetic field has no effect on the flow pattern when $N \leqslant 0.2$ and that a perfectly steady roll structure with axis parallel to the magnetic field is realized when $N \geqslant 2$. Fluctuations of roll-like structure were observed between these two values of $N$. In the present study, $N$ becomes 1.5 for the case in Fig. 2 with spontaneous flow reversals, when substituting the observed horizontal velocity $U=5 \mathrm{~mm} / \mathrm{s}$. This is consistent with the regime diagram, in that a two-dimensional roll structure is dominant but time fluctuations of the pattern are allowed. Without a magnetic field, we observed in this square vessel a more isotropic flow structure with large fluctuations that did not have distinct rolls. In the same way of estimation, $N=2.0$ for the case in Fig. 7(a), and $N=0.9$ for Fig. 7(b). This is also consistent with the regime diagram, in that the Lorentz force is strong enough to stabilize the convective flow in Fig. 7(a), while the inertia force is more dominant and the flow is more turbulent for Fig. 7(b). The geometry of the vessel is also important for the occurrence of flow reversals; we did not observe spontaneous flow reversals in that narrow vessel. Fluctuations of flow pattern in a narrower vessel are limited to induce oscillatory 
behavior of roll-like structure as shown in [17], but fluctuations in a wider vessel can cause global reorganizations of the flow pattern as shown in the present experiments. This may be due to the larger variation of the flow in a wider vessel, such as the emergence of new circulations. Applying a horizontal magnetic field of adequate intensity for a horizontally wide fluid layer may be essential for the occurrence of flow reversals in liquid metal R-B convection. Further investigation with various values of $Q$ is necessary for establishing the regime diagram containing the regime of spontaneous flow reversal in this type of square vessel.

\section{ACKNOWLEDGMENTS}

This work is supported by Grant-in-Aid for Scientific Research No. 21244071 from the Ministry of Education, Culture, Sports, Science and Technology, Japan. The authors express thanks for this support.
[1] S. Chandrasekhar, Hydrodynamic and Hydromagnetic Stability (Oxford University Press, London, 1961).

[2] R. Krishnamurti and L. N. Howard, Proc. Natl. Acad. Sci. USA 78, 1981 (1981).

[3] G. Ahlers, S. Grossmann, and D. Lohse, Rev. Mod. Phys. 81, 503 (2009).

[4] J. J. Niemela, L. Skrbek, K. R. Sreenivasan, and R. J. Donnelly, J. Fluid Mech. 449, 169 (2001).

[5] K. R. Sreenivasan, A. Bershadskii, and J. J. Niemela, Phys. Rev. E 65, 056306 (2002).

[6] X.-L. Qiu and P. Tong, Phys. Rev. E 66, 026308 (2002).

[7] H.-D. Xi, S. Lam, and K.-Q. Xia, J. Fluid Mech. 503, 47 (2004).

[8] C. Sun, K.-Q. Xia, and P. Tong, Phys. Rev. E 72, 026302 (2005).

[9] H.-D. Xi and K.-Q. Xia, Phys. Fluids 20, 055104 (2008).
[10] K. Sugiyama, R. Ni, R. J. A. M. Stevens, T. S. Chan, S.-Q. Zhou, H.-D. Xi, C. Sun, S. Grossmann, K.-Q. Xia, and D. Lohse, Phys. Rev. Lett. 105, 034503 (2010).

[11] T. Segawa, A. Naert, and M. Sano, Phys. Rev. E 57, 557 (1998).

[12] T. Mashiko, Y. Tsuji, T. Mizuno, and M. Sano, Phys. Rev. E 69, 036306 (2004).

[13] Y. Tsuji, T. Mizuno, T. Mashiko, and M. Sano, Phys. Rev. Lett. 94, 034501 (2005).

[14] Y. Takeda, JSME Int. J., Ser. B 38, 8 (1995).

[15] Y. Tasaka, Y. Takeda, and T. Yanagisawa, Flow Meas. Inst. 19, 131 (2008).

[16] T. Yanagisawa, Y. Yamagishi, Y. Hamano, Y. Tasaka, M. Yoshida, K. Yano, and Y. Takeda, Phys. Rev. E 82, 016320 (2010).

[17] T. Yanagisawa, Y. Yamagishi, Y. Hamano, Y. Tasaka, K. Yano, J. Takahashi, and Y. Takeda, Phys. Rev. E 82, 056306 (2010). 